\title{
Multi-task Joint Strategies of Self-supervised Representation Learning on Biomedical Networks for Drug Discovery
}

\section{Xiaoqi Wang}

Hunan University https://orcid.org/0000-0002-5868-5044

\section{Yingjie Cheng}

Hunan University https://orcid.org/0000-0001-7232-9946

\section{Yaning Yang}

Hunan University

Fei Li

Computer Network Information Center, Chinese Academy of Sciences

Shaoliang Peng ( $\nabla$ slpeng@hnu.edu.cn )

Hunan University

\section{Article}

Keywords:

Posted Date: February 9th, 2022

DOI: https://doi.org/10.21203/rs.3.rs-1260249/v1

License: (a) (i) This work is licensed under a Creative Commons Attribution 4.0 International License. Read Full License 


\title{
Multi-task Joint Strategies of Self-supervised Representation
}

\section{Learning on Biomedical Networks for Drug Discovery}

\author{
Xiaoqi Wang ${ }^{1 \dagger}$, Yingjie Cheng ${ }^{1 \dagger}$, Yaning Yang ${ }^{1}, \mathrm{Fei} \mathrm{Li}^{2 *}$, and Shaoliang Peng ${ }^{1,3 *}$ \\ ${ }^{1}$ College of Computer Science and Electronic Engineering, Hunan University, \\ Changsha 410082, China. \\ ${ }^{2}$ Computer Network Information Center, Chinese Academy of Sciences, Beijing \\ 100850, China. \\ ${ }^{3}$ The State Key Laboratory of Chemo/Biosensing and Chemometrics, Hunan University, \\ Changsha 410082, China.
}

†These authors contributed equally to this work.

${ }^{*}$ To whom correspondence should be addressed; E-mail:

pittacus@gmail.com (Fei Li);

and slpeng@hnu.edu.cn (Shaoliang Peng).

\begin{abstract}
Self-supervised representation learning (SSL) on biomedical networks provides new opportunities for drug discovery that lacks available biology or clinic phenotype. However, how to effectively combine multiple SSL models is still challenging and has been rarely explored. Therefore, we propose multi-task joint strategies of selfsupervised representation learning on biomedical networks for drug discovery, named MSSL2drug. We design six basic SSL tasks inspired by various modality features including structures, semantics, and attributes in heterogeneous biomedical networks. In addition, fifteen combinations of multiple tasks are evaluated by a graph attentionbased adversarial multi-task learning framework in two drug discovery scenarios. The results suggest two important findings. (1) Combinations of multimodal tasks achieve the best performance compared to other multi-task joint strategies. (2) The joint training of local and global SSL tasks yields higher performance than random task combinations. Therefore, we conjecture that the multimodal and local-global combination strategies can be treated as a guideline of multi-task SSL for drug discovery.
\end{abstract}

\section{Introduction}

Drug discovery is an important task for improving the quality of human life. However, it is an expensive, time-consuming, and complicated process that has a high chance of failure [1-2]. To improve the efficiency of drug discovery, a great number of researchers have devoted to developing or leveraging deep learning to speed up its intermediate steps, such as molecular property prediction [3-4], drug-target interaction (DTI) predictions [5-8], and drug-drug interaction (DDI) predictions [9-11]. A key advantage behind these methods is that deep learning algorithms can capture the complex 
nonlinear relationships between input and output data [12].

In the past few years, deep learning techniques have gradually emerged as a powerful paradigm for drug discovery. Most deep learning architectures, such as convolutional neural networks [13] and recurrent neural networks [14], operate only on regular grid-like data (e.g., 2D images and text sequences), and are not well suited for graph data (e.g., DDI and DTI networks). However, in the real world, biomedical data are often formed as graphs or networks. In particular, biomedical heterogeneous networks (BioHNs) that integrate multiple types of data source are used extensively for life science researches. This is intuitive since BioHNs are well suited for modeling complex interactions in biological systems. For example, the BioHNs incorporating DDIs, DTIs, and protein-protein interactions (PPIs), protein-disease associations can naturally simulate the 'multi-drugs, multi-targets, multi-diseases' biological processes within human body [11]. In the context of biomedical networks applications, graph neural networks (GNNs) [15-17], which are deep learning architectures specifically designed for graph structure data, are utilized to improve drug discovery. Such studies [18-21] use GNNs to generate the representation of each node in BioHNs, and formulate drug discovery as the node- or edge-level prediction problem. These graph neural network-based drug discovery approaches have shown high-precision predictions. However, most existing methods heavily depend on the size of training sample; that is, only large-scale training samples can help models to achieve great performance, thus leading to poor generalization and weak robustness. Simultaneously, data labeling is expensive and time-consuming. Therefore, these graph-based deep learning models that rely on large-scale labeled data may not be satisfactory in real drug development scenarios.

Self-supervised representation learning (SSL) is a promising paradigm for solving the above issues. In SSL, deep learning models are trained via pretext tasks, in which supervision signals are automatically extracted from unlabeled data without the need for manual annotation. SSL aims to guide models to generate the generalized representations to achieve better performance on various downstream tasks. Following the immense success of SSL on computer vision [22-23] and natural language processing [24-25], SSL models built upon BioHNs have enjoyed increasing attention and have been successfully applied to drug discovery [3, 26-29]. Unfortunately, most existing methods often design a single SSL task to train GNNs for drug discovery, thus leading to the built-in bias toward a single task and ignoring the multi-perspective characteristics of BioHNs. For example, to predict DTIs, DeepR2cov [28] utilizes a masked meta path-driven SSL task that can simultaneously capture the structures and semantics among nodes in BioHNs. However, DeepR2cov ignores the attribute features of nodes. To cope with the potential bottleneck in single task-driven SSL applications, there are a few attempts leveraging multiple SSL tasks for facilitating performance of drug discovery [30-32]. These methods aim to integrate the advantages of various types of SSL tasks via the multi-task learning paradigms. However, most previous approaches train GNNs according to a fixed joint strategy involving multiple tasks, and do not focus on the differences between various multi-task combinations. Concurrently, for a given biomedical heterogeneous network, a variety of potential self-supervised 
tasks and combination strategies can be utilized. However, the determination of which combination strategies can generate the most effective improvements has rarely been explored. Therefore, it is significant to pay attention to the choice of multi-task combination strategies in SSL approaches. In addition, multi-task SSL methods built on biomedical heterogeneous networks for drug discovery are still in the initial stages, and more systematic studies are pressingly needed.

To address the aforementioned problems, we propose the multi-task joint strategies of self-supervised representation learning on biomedical networks for drug discovery, named MSSL2drug. Inspired by three modality features (i.e., structures, semantics, and attributes in BioHNs), six self-supervised tasks are developed to explore the impact of various SSL models on drug discovery. Next, fifteen multi-task joint strategies are evaluated via a graph attention-based adversarial multi-task learning model in two drug discovery scenarios. We find that the combinations of multimodal tasks can generate best performance compared to other multi-task strategies. Another interesting conclusion is that joint training of local and global SSL task tends to yield higher performance than random task combinations.
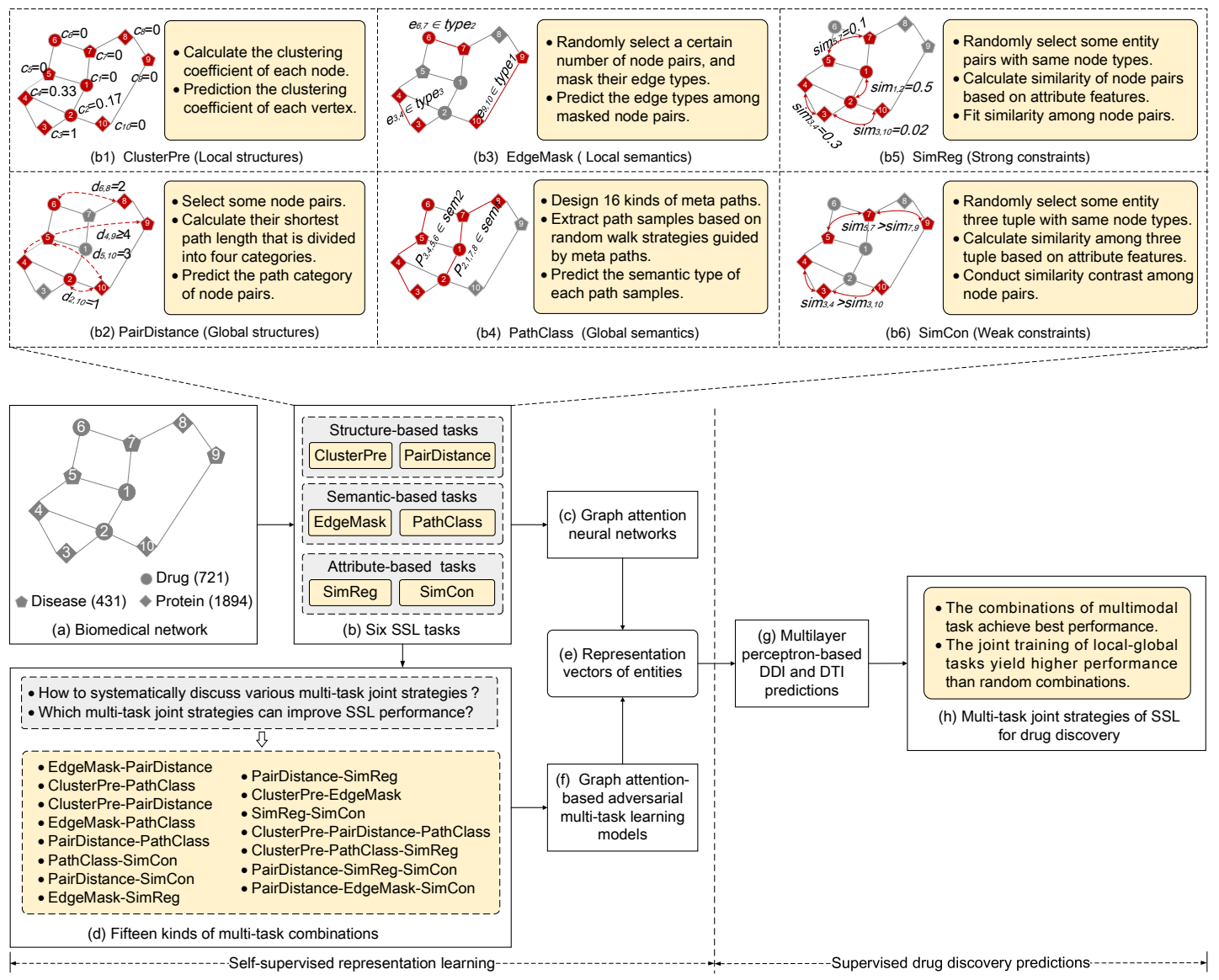

Figure 1. The schematic workflow of MSSL2drug. All circles, quadrangles, and pentagons denote the drugs, proteins, and diseases in BioHNs, respectively. The solid lines are the relationships among the biomedical entities in a BioHN. The red nodes represent the randomly selected vertices or node pairs in each of self-supervised task. The red solid lines in the edge type masked prediction (EdgeMask) and bio-path classification (PathClass) modules represent the randomly selected edges or paths. The dashed curved lines in the pairwise distance classification (PairDistance) module 
represent the measurements of the shortest paths between biomedical entities. The dashed solid lines in the node similarity regression (SimReg) and node similarity contrast (SimCon) modules represent the measurements of the similarities between biomedical entities. ClusterPre and PairDistance denotes clustering coefficient prediction and a pairwise distance classification, respectively.

\section{Result}

\subsection{Overview of MSSL2drug}

As shown in Fig. 1, we propose the multi-task joint strategies of self-supervised representation learning on biomedical networks for drug discovery, named MSSL2drug. First, we construct a biomedical heterogeneous network that integrates 3,046 biomedical entities and 111,776 relationships. Second, we develop six self-supervised tasks based on structures, semantics, and attributes in the BioHN, as shown in Fig. 1 b. These self-supervised tasks guide graph attention networks (GATs) to generate the representations from different views in the BioHN. More importantly, we develop fifteen kinds of multi-task combinations and a graph attention-based adversarial multitask learning framework to improve the representation quality. After completing selfsupervised representation learning, the different representations from single task and multi-task SSL are fed into a multilayer perceptron (MLP) for predicting DDIs and DTIs. Based on the experiment results, we can draw two important findings. (1) The combinations of multimodal SSL tasks achieve state-of-the-art performance of drug discovery. (2) The joint training of local and global SSL tasks is superior to the random combination of two SSL tasks, and contributes to improving drug discovery predictions.
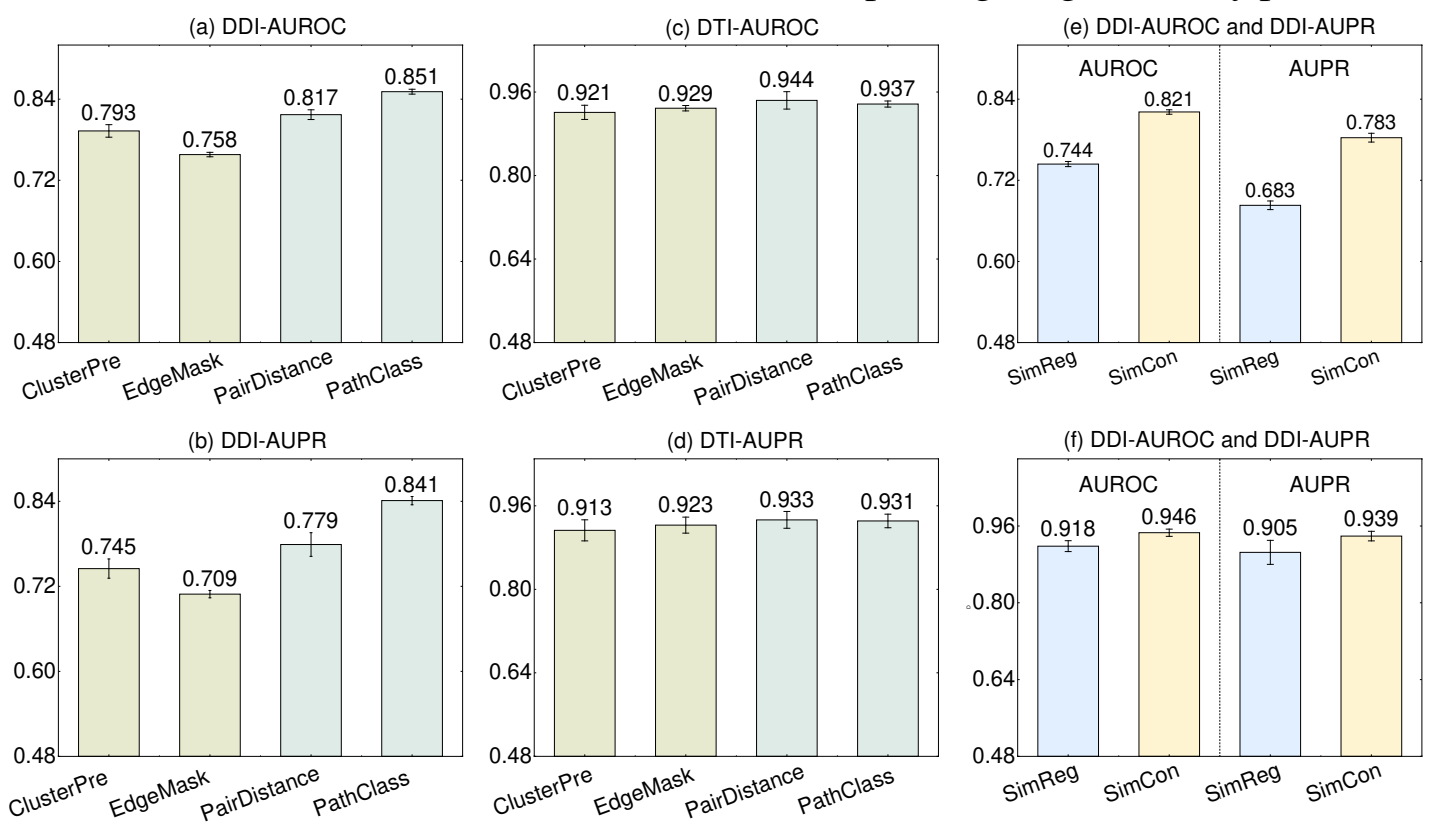

$\square$ Local information-based SSL

Gloabl information-based SSL

$\square$ Strong constraint-based SSL $\quad \square$ Weak constraint-based SSL

Figure 2. The results of single SSL tasks for warm start drug predictions, where the area under precision recall (AUPR) curve and area under receiver operating characteristic (AUROC) curve is used for the evaluation metrics. The error bars denote standard deviation values that are calculated across ten results. 


\subsection{Performance of single task-driven SSL on drug warm start predictions}

The low-dimensional representation vectors derived from SSL are applied to DTI predictions and DDI predictions that play important roles in drug discovery. The results obtained by SSL on warm start drug predictions are shown in Fig. 2. Intuitively, we can observe that PathClass (DDI-AUROC $=0.851$, DDI-AUPR $=0.841$, DTI-AUROC $=$ 0.937, DTI-AUPR=0.931) attains better performance than other SSL methods. Subsequently, we find that PairDistance and PathClass significantly outperform ClusterPre and EdgeMask. In particular, PathClass attains approximately 10-15\% improvements over EdgeMask in terms of AUROC and AUPR scores for DDI and DTI predictions. Another aspect to note is that SimCon is superior to SimReg, with approximately $12.5 \%$ average improvements for DDI predictions. These results suggest that the global information-driven SSL approaches are superior to the local informationbased SSL tasks. In addition, attribute weak constraint-based SSL tasks are superior to strong constraint-based models.

Table 1. Performances of two-task combinations for warm start drug predictions.

\begin{tabular}{|c|c|c|c|c|c|c|}
\hline \multirow{2}{*}{ No. } & \multirow{2}{*}{$\begin{array}{l}\text { Combination of SSL } \\
\text { tasks }\end{array}$} & \multirow{2}{*}{ Category } & \multicolumn{2}{|c|}{ DDI } & \multicolumn{2}{|c|}{ DTI } \\
\hline & & & AUROC \pm std & AUPR \pm std & AUROC \pm std & AUPR \pm std \\
\hline 1 & EdgeMask-PairDistance & L-G & $\mathbf{0 . 9 1 7} \pm 0.003$ & $\mathbf{0 . 9 1 2} \pm 0.005$ & $\mathbf{0 . 9 5 8} \pm 0.007$ & $0.951 \pm 0.009$ \\
\hline 2 & ClusterPre-PathClass & L-G & $0.915 \pm \mathbf{0 . 0 0 1}$ & $0.910 \pm \mathbf{0 . 0 0 3}$ & $0.956 \pm 0.007$ & $0.956 \pm 0.007$ \\
\hline 3 & ClusterPre-PairDistance & L-G & $0.895 \pm 0.002$ & $0.882 \pm 0.004$ & $0.945 \pm 0.006$ & $0.936 \pm 0.011$ \\
\hline 4 & EdgeMask-PathClass & L-G & $0.882 \pm 0.009$ & $0.869 \pm 0.011$ & $0.946 \pm \mathbf{0 . 0 0 5}$ & $0.933 \pm 0.007$ \\
\hline 5 & PairDistance-PathClass & G-G & $0.887 \pm 0.004$ & $0.871 \pm 0.005$ & $0.943 \pm 0.006$ & $0.937 \pm 0.007$ \\
\hline 6 & PathClass-SimCon & G-W & $0.883 \pm 0.004$ & $0.867 \pm 0.006$ & $0.947 \pm 0.009$ & $0.945 \pm 0.010$ \\
\hline 7 & PairDistance-SimCon & G-W & $0.880 \pm 0.006$ & $0.860 \pm 0.012$ & $0.942 \pm 0.007$ & $0.935 \pm 0.008$ \\
\hline 8 & EdgeMask-SimReg & L-S & $0.875 \pm 0.004$ & $0.856 \pm 0.009$ & $0.946 \pm 0.006$ & $0.932 \pm 0.011$ \\
\hline 9 & PairDistance-SimReg & $\mathrm{L}-\mathrm{S}$ & $0.873 \pm 0.004$ & $0.847 \pm 0.010$ & $0.936 \pm 0.005$ & $0.924 \pm 0.008$ \\
\hline 10 & ClusterPre-EdgeMask & L-L & $0.863 \pm 0.003$ & $0.839 \pm 0.007$ & $0.938 \pm 0.007$ & $0.928 \pm 0.012$ \\
\hline 11 & SimReg-SimCon & S-W & $0.858 \pm 0.002$ & $0.836 \pm 0.003$ & $0.916 \pm 0.010$ & $0.915 \pm 0.013$ \\
\hline
\end{tabular}

(a) 'L' and 'G' denote the local and global information-based SSL tasks, respectively. 'W' and 'S' denote the weak constraint and strong constraint-based SSL tasks, respectively.

(b) 'std' denotes the standard deviation value calculated across ten results.

(c) The best results are marked in boldface.

\subsection{Achieve superior performance by joint training the local and global SSL tasks in warm start scenarios}

Based on a graph attention-based adversarial multi-task learning framework, we compare and discuss various combinations of multiple self-supervised tasks for drug discovery. The results obtained with ten combinations of tasks are shown in Table 1. Although PairDistance and SimCon generate great results, we find that PairDistanceSimCon shows the unsatisfactory performance (DDI-AUROC $=0.848$, and DTIAUROC $=0.913$ ). In contrast, EdgeMask-PairDistance (DDI-AUROC=0.917, DTIAUROC $=0.958$ ) and ClusterPre-PathClass (DDI-AUROC $=0.915$, DTI-AUROC $=$ 
0.956) produce relatively high results, with 2.0-5.4\% higher AUROC and 2.8\%-7.5\% higher AUPR than other task combinations for DDI predictions. Concurrently, ClusterPre-PairDistance and EdgeMask-PathClass also produce promising results on DTI and DDI predictions. More interestingly, we find that EdgeMask-PairDistance, ClusterPre-PathClass, ClusterPre-PairDistance and EdgeMask-PathClass are the combinations of global and local SSL tasks. In addition, PairDistance-SimCon (DDIAUPR=0.860, DTI-AUPR=0.935) is superior to PairDistance-SimReg (DDI-AUPR= 0.847, DTI-AUPR=0.924). Similar situations are observed when we compare SimCon and SimReg. The results in Fig. 3 further suggest that the joint training of local and global SSL tasks tends to obtain higher performance than random combinations of two SSL tasks. Therefore, we conjecture that the local-global combination strategy can be regarded as an effective guideline for multi-task SSL to drug discovery.

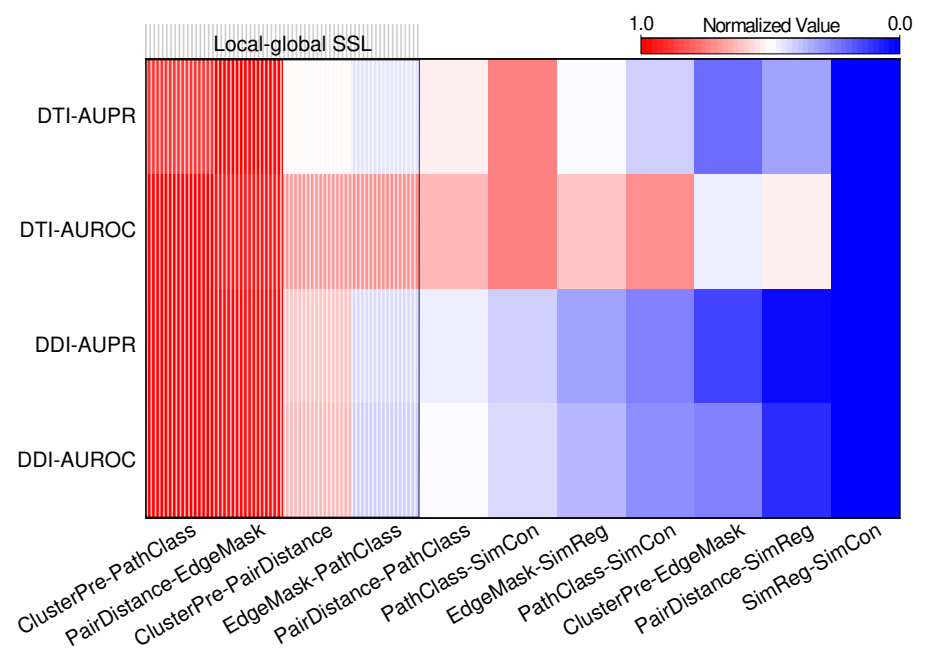

Figure 3. Heatmap of ten kinds of two-task combinations for warm start drug predictions where the results are normalized along the $\mathrm{x}$-axis. The redder (bluer) squares denote the greater (smaller) the value. The shaded area denotes the combinations of global and local SSL tasks.

\subsection{Achieve state-of-the-art performance by combining multimodal SSL tasks in warm start scenarios}

EdgeMask-PathClass is a combination of local-global SSL tasks, as shown in Table 1. However, there is no significant difference between EdgeMask-PathClass and PairDistance-PathClass. We also observe that a similar phenomenon occurs in the comparisons between EdgeMask-PathClass and PathClass-SimCon. This may be attributed to the fact that EdgeMask-PathClass includes only single type of modality information (i.e., the structures of BioHNs). However, PairDistance-PathClass and PathClass-SimCon capture two modalities of information (i.e., the structures and semantics of BioHNs). These results seem to indicate that we should consider the effects of different modalities. Therefore, we further design four combinations of multimodal tasks that refer to the structures, semantics and attributes of BioHNs.

The results obtained with fifteen multi-task combinations are listed in the Supplementary Materials Table S3. We find that the top two combination models are ClusterPre-PathClass-SimReg and PairDistance-EdgeMask-SimCon. Interestingly, 
they include three modalities of information, i.e., structure, semantic and attribute knowledge. Although EdgeMask-PathClass and EdgeMask-PairDistance belong to the local-global task combinations, as shown in Table 1 and Fig. 4a, EdgeMaskPairDistance is superior to EdgeMask-PathClass, with DDI-AUROC and DDI-AUPR improvements of approximately $3.5 \%$ and $4.3 \%$, respectively. Similar phenomena is observed between ClusterPre-PathClass (DDI-AUROC $=0.915$, DDI-AUPR $=0.910$ ) and ClusterPre-PairDistance (DDI-AUROC=0.895, DDI-AUPR=0.882). In other words, the combinations of two-modal tasks (e.g., EdgeMask-PairDistance and ClusterPre-PathClass) generate better results than the combinations of single-modal tasks (e.g., EdgeMask-PathClass and ClusterPre-PairDistance). The results in Fig. 4b and c show the same situations; that is, the growth of modalities lead to the performance improvement for drug discovery. More interestingly, we notice that PairDistanceSimReg-SimCon has one more task than PairDistance-SimReg. However, its DTI prediction performance exhibits no significant improvement. In contrast, for DDI predictions, PairDistance-SimReg-SimCon leads to a slight reduction compared to PairDistance-SimReg. This may be because PairDistance-SimReg-SimCon, in which the three tasks have only two-modal views (i.e., structures and semantics information), fails to increase the number of modalities over that used by PairDistance-SimReg and generates some noise. Similarly, ClusterPre-PairDistance-PathClass and ClusterPrePathClass exhibit the same trends and phenomena. These results suggest that combinations of multimodal tasks can achieve best representation performance for drug discovery. Therefore, we conjecture that the multimodal combination strategy can be regarded as a potential guideline for multi-task SSL on drug discovery.

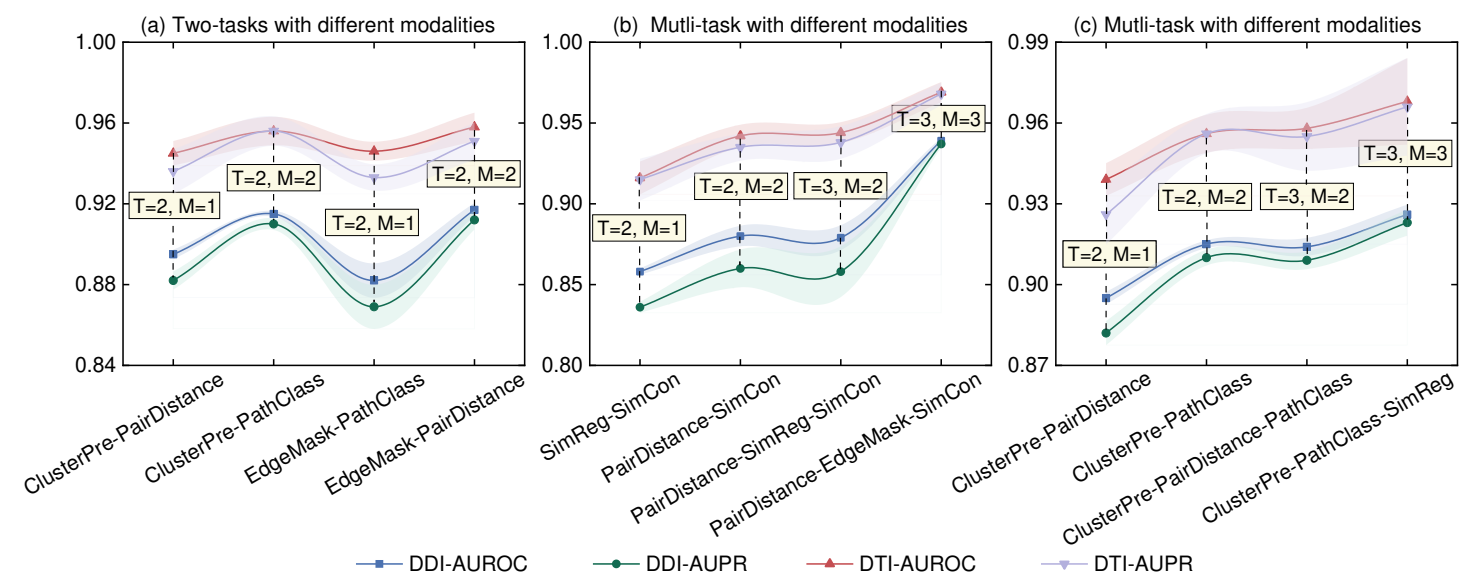

Figure 4. The results obtained with multimodal task combinations for drug predictions, where ' $M$ ' and ' $\mathrm{T}$ ' denote the total number of tasks and modalities in each multi-task combination, respectively.

\subsection{The results of cold start predictions further demonstrate the influence of local- global and multimodal combination strategies on drug discovery}

To further validate the conclusions found in the warm start drug prediction scenarios, we simulate the real drug discovery; that is, we predict the potential targets and drugs that may interact with newly discovered chemical compounds. Similarly, the results of DDI and DTI predictions are generated by six basic SSL tasks and fifteen kinds of 
multi-task combinations (as shown in the Supplementary Materials Table S4).
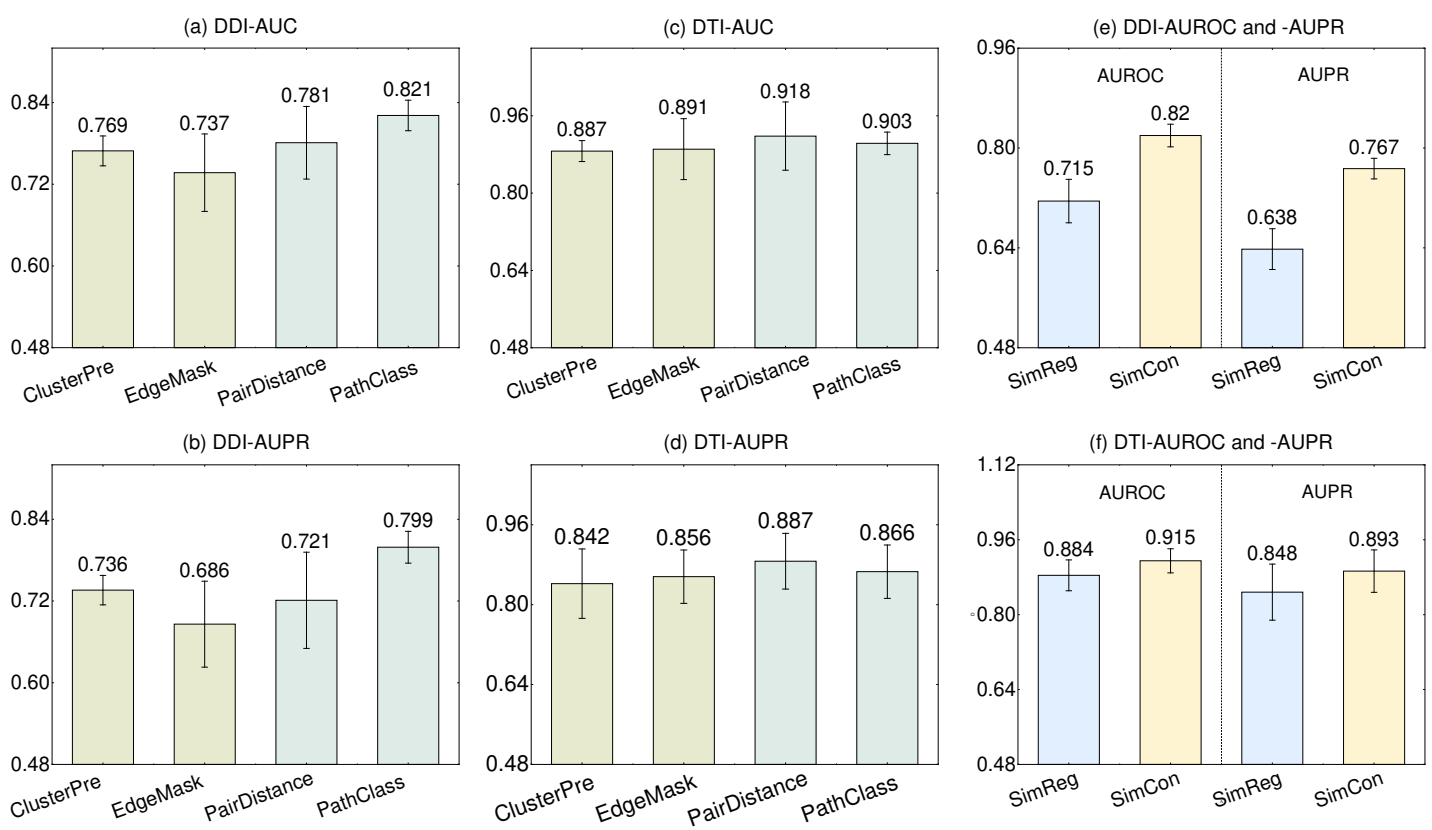

Local information-based SSL

Gloabl information-based SSL

$\square$ Strong constraint-based SSL

$\square$ Weak constraint-based SSL

Figure 5. The results of single SSL tasks for cold start predictions.

The results obtained with the single SSL tasks are shown in Fig. 5. As mentioned above, PairDistance and PathClass yield better results than ClusterPre and EdgeMask. In particular, PathClass outperforms EdgeMask with $8.4 \%$ and $11.3 \%$ improvements in terms of AUROC and AUPR for DDI predictions, respectively. These results are straightforward and effective demonstrations that global information-based SSL can achieve better performance than local information-based SSL. Similarly, SimCon is superior to SimReg further suggesting that the attribute weak constraint-based SSL tasks outperform the strong constraint-based tasks.

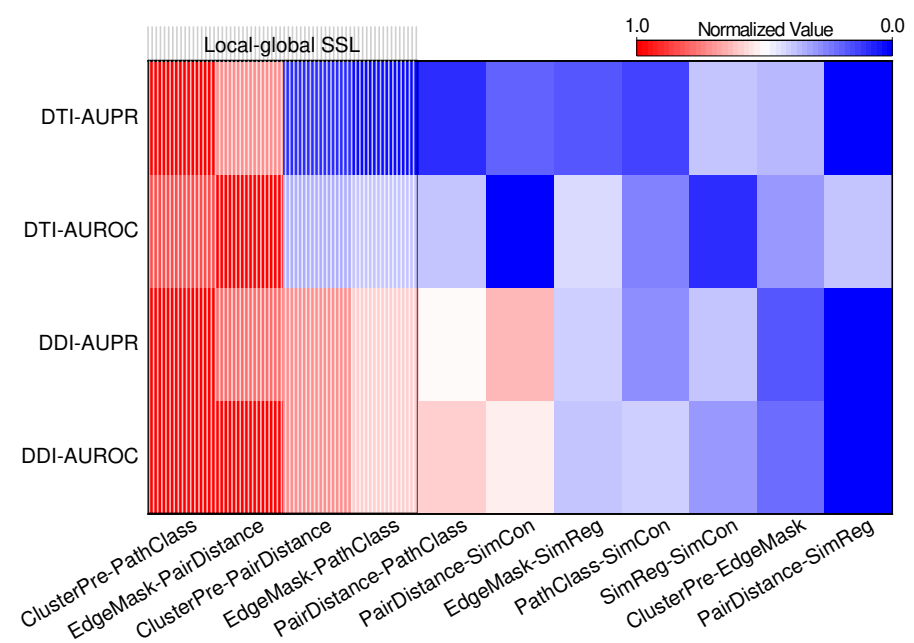

Figure 6. Heatmap of ten kinds of two-task combinations for cold start predictions where the results are normalized along the $\mathrm{x}$-axis.

In the two-task combinations scenarios, as shown in Fig. 6, we observe the same phenomena as those exhibited in the cold start for drug scenarios: the top three models 
are the local-global SSL tasks, that is, ClusterPre-PathClass (DDI-AUROC $=0.889$, DTI-AUROC $=0.927$ ), EdgeMask-PairDistance (DDI-AUROC $=0.890$, DTI-AUROC $=$ 0.923), and ClusterPre-PairDistance (DDI-AUROC $=0.871$, DTI-AUROC $=0.911$ ). These results further certify the local-global combination strategy that the local and global SSL tasks jointly guide GNNs to generate superior drug discovery predictions.

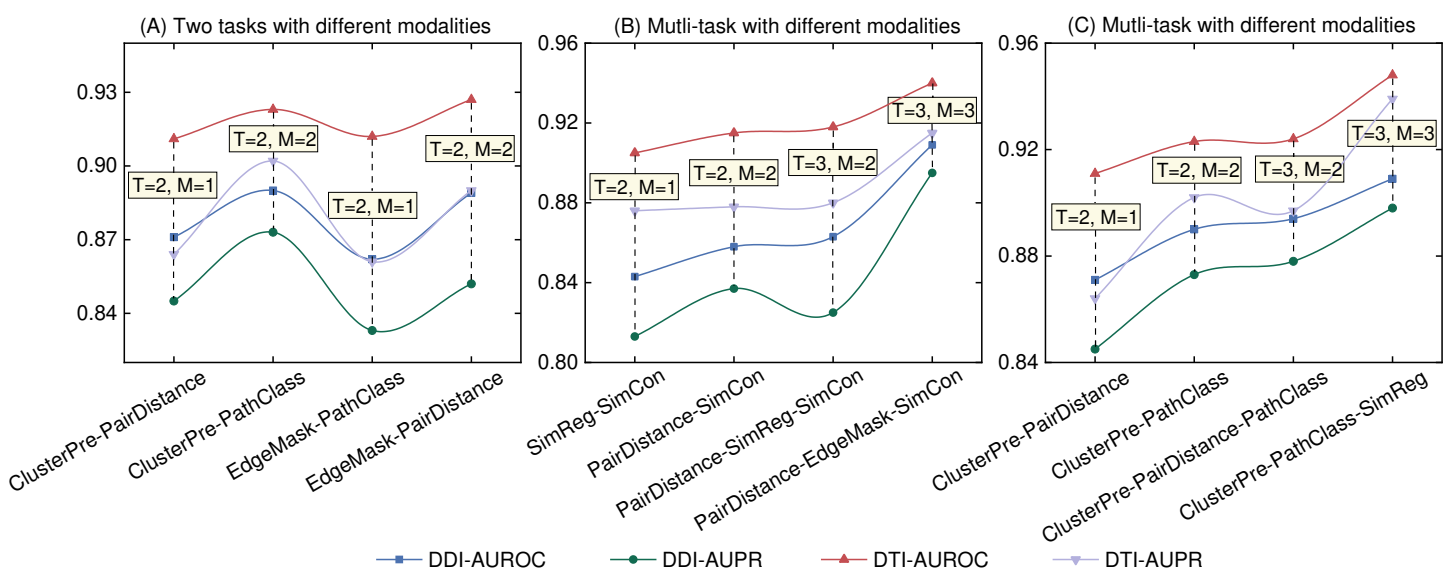

Figure 7. The results obtained with multimodal SSL tasks for cold start drug discovery, where ' $M$ ' and ' $\mathrm{T}$ ' denote the total number of tasks and modalities in each multi-task combination, respectively.

In addition, the results of the multimodal tasks are shown in Fig. 7. We find that the SSL task combinations containing three modalities of information, such as ClusterPre-PathClass-SimReg (DDI-AUROC $=0.909, \quad$ DTI-AUROC $=0.948$ ) and PairDistance-EdgeMask-SimCon (DDI-AUROC $=0.909$, DTI-AUROC $=0.940$ ), are superior to the task combinations capturing two-modal knowledge, such as ClusterPrePairDistance-PathClass (DDI-AUROC $=0.894, \quad$ DTI-AUROC $=0.924), \quad$ and PairDistance-SimReg-SimCon (DDI-AUROC $=0.863, \quad$ DTI-AUROC $=0.918$ ). Concurrently, the two-modal SSL task combinations outperform the one-modality SSL task combinations. In other words, as the number of modalities increases, the performance of cold start predictions is improved. These results further verify the multimodal combination strategy, that is, combinations of multimodal SSL tasks can achieve state-of-the-art the prediction performance of drug discovery.

\section{Discussion}

Recently, self-supervised representation learning on biomedical heterogeneous networks has emerged as a promising paradigm for drug discovery. However, the determination of which combination strategies can generate the most effective improvements is challenging and rarely explored. Therefore, we propose is a unified combination strategy of multi-task self-supervised learning on biomedical heterogeneous networks for drug discovery. First, this work develops six selfsupervised learning tasks that are based on the various modality features including structures, semantics, and attributes in BioHNs. These tasks are used to train graph attention networks for DDI and DTI predictions. The results show that global knowledge-based SSL models outperform local information-based SSL models for 
drug discovery. This is intuitive and understandable since global view-based SSL tasks can capture the complex structures and semantics that is unable to be naturally learned by local SSL models. We also find that attribute weak constraint-based SSL tasks are superior to strong constraint-based models. This may be attributed to the fact that the utilized similarity scoring functions are handcrafted and unable to accurately reflect the similarities among nodes. Unfortunately, the node similarity regression tasks arbitrarily fit node similarity values of all node pairs. In contrast, similarity contrast tasks reduce the dependence on the original feature similarity values. Therefore, the global selfsupervised information in BioHNs has more potential and can help enhance the drug discovery. Concurrently, we can preferentially select an attribute weak constraint-based SSL model for drug discovery when the similarity scoring functions fail to guarantee precise node similarity evaluations in the original feature space.

More importantly, fifteen kinds of multiple task combinations are evaluated by a graph attention-based adversarial multi-task learning model for drug discovery. These results suggest that the joint training the global and local tasks can achieve the relatively high DDI and DTI prediction performances. In contrast, combining tasks with great performance does not necessarily lead to better performance than other multi-task combinations for drug discovery. This is intuitive since there may be some conflicts and redundancies in the random combinations of SSL tasks. However, the combinations of global and local SSL strategies enable GNNs to leverage complementary information in BioHNs. In other words, the local graph SSL models can capture the features within node itself or its first-order neighbors, but ignore the bird's-eye view of the node position in BioHNs. Fortunately, global SSL models can learn the dependencies among long-range neighborhoods, thus compensating for the shortcomings of local SSL tasks.

Simultaneously, an interesting finding is that combinations with multimodal tasks tend to generate state-of-the-art representation performance, thus leading to improvements of drug discovery predictions. This is because the combinations of multimodal tasks can capture multi-view information including structure, semantic and attribute features in BioHNs. Better representations can be generated when constraints derived from different modalities are available during the training process. The multimodal SSL models allow for knowledge transfer across multiple views and attain a deep understanding of natural phenomena in BioHNs. To enable SSL to further promote drug discovery predictions, multimodal tasks need to be combined to leverage the complementarity of heterogeneous data and provide more robust predictions.

In conclusion, self-supervised representation learning based on biomedical heterogeneous networks provides new opportunities for drug discovery. To facilitate this line of research, we carefully explore the influence of various basic SSL tasks and propose unified combination strategies involving multi-task self-supervised representation learning to improve drug discovery. Simultaneously, we present a detailed empirical study to understand which combination strategies of multiple SSL tasks are most effective for drug discovery. In the future, we will further verify the proposed global-local and multimodal combination strategy on more BioHNs, and expand their application scope. In addition, we will pay attention to designing more SSL tasks and combination strategies to further improve performance of drug discovery. 


\section{Materials and methods}

\subsection{Biomedical heterogeneous networks}

In this work, we construct a biomedical heterogeneous network according to deepDTnet [33]. The constructed BioHN assembles three types of nodes (i.e., drugs, proteins and diseases) and five types of edges (drug-drug interactions, drug-protein interactions, drug-disease associations, and protein-disease associations). The BioHN contains 3,046 nodes and 111,776 relationships. The detailed construction processes of the BioHN can be found in the Supplementary Material Section S1.

\subsection{Basic self-supervised learning tasks}

Multimodal information, including the structures, semantics, and attributes in BioHNs, provides unprecedented opportunities for designing advanced self-supervised pretext tasks. Hence, we develop six self-supervised tasks based upon the multimodal information contained in BioHNs for drug discovery.

\subsubsection{Structure-based SSL tasks}

The first direct choice for constructing SSL tasks is the inherent structure information contained in BioHNs. For a given node, self-supervision information is not only limited to itself or local neighbors, but also includes a bird's-eye view of the node positions in a BioHN. Therefore, we design a clustering coefficient prediction (ClusterPre) that captures local structures and a pairwise distance classification (PairDistance) that reflects the global structure information in BioHNs.

Clustering coefficient prediction (ClusterPre): In this pretext task, we use GNNs to predict the clustering coefficient [34] of each vertex in a BioHN. The ClusterPre SSL task aims to guide GNNs to generate low-dimensional representations that preserve the local structure information in the BioHN. In ClusterPre, the loss function adopts the mean squared error (as described in Supplementary Material Section S2.1).

Pairwise distance classification (PairDistance): We develop PairDistance that is not limited to a node itself and its local neighborhoods; it also takes global views of a BioHN. Similar to $S^{2}$ GRL [35], we randomly select a certain number of node pairs and calculate the shortest path length between each node pair $(i, j)$ as its distance value $d_{i, j}$. Subsequently, these node pairs and distance values are used to train GNNs for drug discovery. In practice, the distances between all node pairs are divided into four categories, that is, $d_{i, j}=1, d_{i, j}=2, d_{i, j}=3$ and $d_{i, j} \geq 4$. In other words, the PairDistance SSL task can be treated as a multiclass classification problem for which we adopt the cross entropy loss function (as described in the Supplementary Material Section S2.2). This is mainly attributed to two reasons. (1) The distinctions between the node node pairs interacting via longer paths (i.e., $d_{i, j} \geq 4$ ) are relatively vague; thus, it 
is more reasonable to divide the longer pairwise distances into one "major" class. (2) Based on the small-world phenomenon [36], we suppose that the shortest path lengths between most node pairs are within a certain range. If we fit longer pairwise distances, some noisy values will be generated. Here, $d_{i, j} \geq 4$ indicates that PairDistance is not limited to the local connections in the BioHNs. Therefore, PairDistance is beneficial for guiding GNNs to generate node representation vectors that encode the global topology information of the BioHNs.

\subsubsection{Semantic-based SSL tasks}

BioHNs integrates multiple types of nodes or edges. The different relationships among these nodes contain distinct semantic information. Recent studies have suggested that semantic information can contribute to learning high-quality representations [28, 31]. Therefore, we develop edge type masked prediction (EdgeMask) and bio-path classification (PathClass) tasks for encouraging GNNs to capture certain aspects of semantic knowledge. Similar to the structure-based SSL tasks, EdgeMask and PathClass can capture the local and global semantics of BioHNs, respectively.

Edge type masked prediction (EdgeMask): This task is inspired by the BERT model [24], in which the core is a masked language model [37]. More specifically, we randomly mask edge types among some node pairs and then use GNNs to predict these edge types, where the edge representations are obtained by concatenating the representations of their two end-nodes. A detailed description of EdgeMask is found in Supplementary Materials Section 1.3. The types of edges indicate the different action mechanisms between biomedical entities. Therefore, EdgeMask can enable GNNs to learn the semantic features among local neighborhoods.

Bio-meta path classification (PathClass): Compared to the types of edges among nodes, meta paths (as described in the Supplementary Materials Section S1.4) are a sequences for incorporating the complex semantic relationship in BioHNs. Different types of meta paths indicate distinct semantics. In PathClass, we design 16 types of meta paths as shown in the Supplementary Materials Table S1, where the first or last objects are drugs or proteins, respectively. This is mainly because drugs and proteins are interconnected with other entities by more edges [28]. These meta paths guide random walks to extract path samples from BioHNs. In addition, we generate an equal number of false path instances by randomly replacing some nodes in each true path instance. Therefore, all path samples are divided into 17 categories, including 16 kinds of true meta paths and one kind of false meta paths. Finally, we use GNNs to predict the type of each path sample for learning node representations that contain rich semantic and complex relationships. Similar to PairDistance, we adopt the cross entropy as loss function in PathClass.

\subsubsection{Attribute-based SSL tasks}

In addition to structures and semantics, attribute features play key roles in selfsupervised representation learning. More generally, nodes with similar properties, such as the simplified molecular input line entry system (SMILES) strings [38] of drugs, 
should be distributed closely in the representation space. However, GNNs only aggregate the features of the nodes themselves and their local neighborhoods, thus losing the similarity features among nodes. Based on this intuition, we develop two attribute-based SSL tasks, i.e., node similarity regression (SimReg) and node similarity contrast (SimCon), to enable GNNs to maintain the similarity attributes in the original feature space. According to the degree of dependence on the original feature similarities, SimReg and SimCon can be categorized as strong constraint- and weak constraintbased SSL paradigms, respectively.

Node similarity regression (SimReg): The proposed SimReg task requires GNNs to fit similarity distributions of node pairs. More specifically, we randomly select a certain number of node pairs $(i, j)$ (where $i$ and $j$ are the same types of nodes); and then calculate their similarity value $\operatorname{sim}_{i, j}$ in the original feature space, such as the similarity between SMILES sequences. We require that the cosine values between the embedding vectors generated by the GNNs are close to their similarity values $\operatorname{sim}_{i, j}$ as possible. In other words, SimReg encourages the GNNs to learn representations via a strong constraint-based SSL paradigm. In this work, we use different property similarity measurements according to the various types of nodes. The Tanimoto coefficient [39] among the SMILES sequences of drugs are treated as drugdrug similarity scores. We leverage the Smith-Waterman algorithm [40] to calculate the sequence similarity scores of protein pairs. The disease similarity scores are obtained by using the PPI-based ModuleSim algorithm [41]. The detailed similarity measurement approaches and objective functions are described in Supplementary Materials Section 1.5.

Node similarity contrast (SimCon): In SimReg, the similarity scoring mechanisms have an important impact on the representation learning process. SimReg cannot guarantee to generate the high-quality representations when the node similarity scores in the original feature space may not accurately reflect the true similarity. Therefore, we propose SimCon to reduce the influence of similarity scoring mechanisms. In SimCon, it assumes that the similar nodes in the original features should be closer in the embedding space than dissimilar nodes. More specifically, we randomly select a certain number of three tuples $(i, j, k)$ for nodes, where $i, j$ and $k$ belong to the same types of nodes and $\operatorname{sim}_{i, j} \geq \operatorname{sim}_{i, k}$. For a given tuple $(i, j, k)$, we use GNNs to conduct a node similarity contrast; that is, the cosine values $\left(\cos _{i, j}\right.$ and $\cos _{i, k}$ ) between the node representations generated by the GNNs should satisfy $\cos _{i, j} \geq \cos _{i, k}$. Formally, we propose a novel objective function: 


$$
\ell_{\text {simCon }}(\theta)=\frac{1}{|M|} \sum_{(i, j, k) \in M} L(i, j, k)
$$

where $M$ is the selected set of three tuples $(i, j, k),|M|$ is the number of three tuple, and $L(i, j, k)$ is calculated as follows:

$$
L(i, j, k)= \begin{cases}0, & g(i, j, k) \geq 0 \\ g(i, j, k) & \text { otherwise }\end{cases}
$$

where $g(i, j, k)$ is calculated as follows:

$$
g(i, j, k)=\left(\cos \left(f_{\theta}(i), f_{\theta}(j)\right)-\cos \left(f_{\theta}(i), f_{\theta}(k)\right)\right)-\left(\operatorname{sim}_{i, j}-\operatorname{sim}_{i, k}\right)
$$

where $\theta$ is the parameters of a graph neural network $f_{\theta}(\cdot)$ and $f_{\theta}(i)$ denotes the embedding of node $i$. In addition, $\cos (\cdot, \cdot)$ is the cosine similarity value between two embedding vectors.

Obviously, SimCon only requires that the GNNs can distinguish the similarity between node pairs $(i, j)$ and node pairs $(i, k)$. However, SimReg requires that the GNNs fit similarity distributions for node pairs. Therefore, SimCon reduces the dependence on the original feature similarity values relative to that of SimReg task; thus, SimCon is a weak constraint-based SSL paradigm.

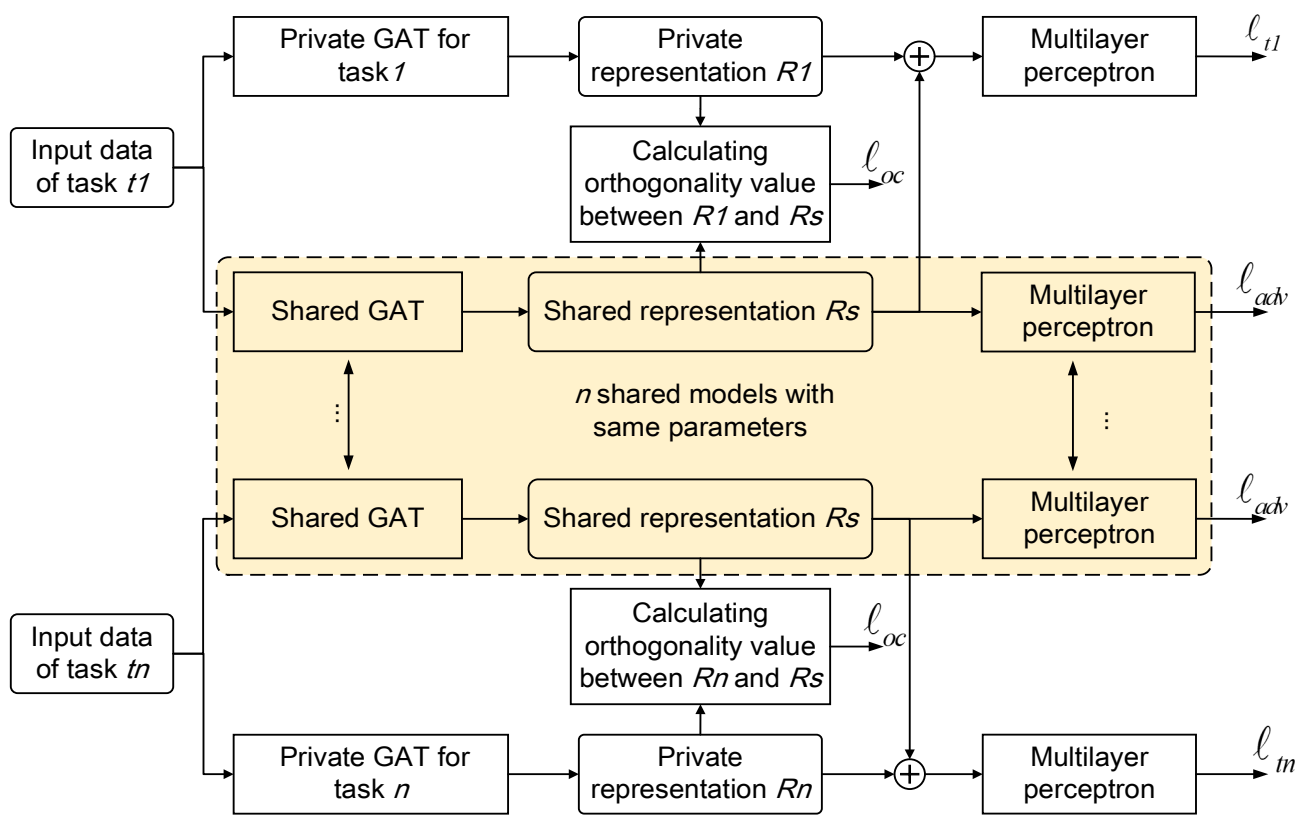

Figure 8. The framework of graph attention-based adversarial multi-task learning. 


\subsection{Graph attention-based adversarial multi-task learning}

In this work, the integration of the multi-task learning and GNNs is a challenging and critical problem. Therefore, we propose a graph attention-based adversarial multi-task learning framework for drug discovery, as shown in Fig. 8. The graph attention-based adversarial multi-task learning framework can be divided into the private and share parts that employ graph attention networks (GATs) [16] with different parameters. The private GAT model generates task-specific representations to make accurate predictions. In contrast, the representations obtained from the shared GAT are fed into a task discriminator that aims to evaluate what kind of task the shared representations come from. Inspired by [42], the shared GAT is trained by an adversarial learning mechanism to ensure that the shared feature space simply contains common and taskinvariant information.

\subsubsection{Graph attention network}

The graph attention network (GAT) [16] is a popular graph neural network. GAT assumes that the contributions of neighboring nodes to the central nodes are different. Therefore, GAT calculates the embedding of each node by aggregating its neighbor features with the learned weights that are dynamically attained by an attention mechanism during the training process.

\subsubsection{Task discriminator}

For any node $i$ in task $t$, the shared GAT generates task-invariant representations $x_{t}^{i}=f_{\theta_{s}}(i)$ where $\theta_{s}$ is the parameter of the shared GAT $f_{\theta_{s}}(\cdot)$. Then, these representation vectors $x_{t}^{i}$ are fed into a multilayer perceptron (MLP) that is treated as the task discriminators. This multilayer perceptron aims to predict what kind of task the shared representation vectors come from.

$$
D\left(x_{t}^{i}, \theta_{t d}\right)=\operatorname{softmax}\left(\operatorname{MLP}_{\theta_{t d}}\left(x_{t}^{i}\right)\right)
$$

where $\operatorname{MLP}(\cdot)$ is a multilayer perceptron in which the trainable parameter is $\theta_{t d}$.

\subsubsection{Adversarial learning mechanism}

In this work, an adversarial mechanism guides a model to generate shared representations such that a task discriminator cannot reliably recognize which task the input representations come from. More specially, given a node, the shared GAT aims to generate a representation to mislead the task discriminator. However, the discriminator makes efforts to correctly determine which task the node representations come from. The adversarial loss can be calculated as follows:

$$
\ell_{a d v}=\min _{\theta_{s}}\left(\max _{\theta_{t d}} \sum_{t=1}^{T} \sum_{i=1}^{N_{t}} y_{t}^{i} \log \left[D\left(f_{\theta_{s}}(i), \theta_{t d}\right)\right]\right)
$$

where $N_{t}$ is the number of training nodes in task $t$, and $y_{t}^{i}$ denotes the ground-truth 
label indicating the type of the current task.

After sufficient training, the shared GAT and task discriminator reach a balance, at which they cannot be improved. Therefore, the shared GAT generates representations that are not contaminated by task-specific features.

\subsubsection{Orthogonality constraints}

The above model generates some task-invariant features that may appear in both the shared space and private space. Therefore, we adopt orthogonality constraints [43] to eliminate redundant features from the private and shared spaces. Formally, the objective function of the orthogonality constraints is calculated as follows:

$$
\ell_{o c}=\sum_{i=1}^{T} \sum_{i=1}^{N_{t}}\left\|f_{\theta_{t}}(i)^{\mathrm{T}} \cdot f_{\theta_{s}}(i)\right\|_{F}^{2}
$$

where $\|\cdot\|_{F}^{2}$ is the squared Frobenius norm, and $f_{\theta_{t}}(\cdot)$ is the private GAT of the current task $t$.

\subsubsection{Multi-task training}

The final loss function of multi-task SSL can be written as follows:

$$
\ell_{\text {total }}=\ell_{t}+\lambda \ell_{a d v}+\gamma \ell_{o c}
$$

where $\lambda$ and $\gamma$ are hyperparameters. $\ell_{t}$ denotes the loss value of task $t$.

During multi-task learning phase, inspired by [44], the models are trained jointly in a stochastic manner by looping over the tasks.

Step 1: Select the next task.

Step 2: Sample an epoch of instances from the task and feed-forward the neural network.

Step 3: Update the corresponding parameters by back-propagation.

Step 4: Go to Step 1.

In this way, each private and shared GAT model is updated by the corresponding specific task. However, all shared GAT models utilize the same parameters. In other words, we attain multiple private GAT models and a shared GAT model.

\subsection{Experiment set}

In SSL stage, we adopt the Glorot initialization [45], the Adam optimizer [46] with a learning rate 5e-4, L2 regularization 5e-4, 8 hidden units and 8 head attentions. The number of epoch is set to 10. In supervised drug discovery, an MLP with three fully connected layers (including an input layer, a hidden layer and an output layer) is used to decode the embedding vectors. The size of the input layer depends on the dimensionality of the input feature, and the size of the hidden layer is set to 64 . We also use the Adam optimizer to train the MLP for 30 epochs with batch size 128. For the learning rate, we select 10 points that are equidistant from the interval $[5 e-4,1]$ and 
then select the best learning rate. We use the area under precision recall (AUPR) curve and area under receiver operating characteristic (AUROC) curve as the evaluation metrics for drug discovery. Each model is executed 10 times, and the average performance is computed.

In this study, we focus on the performance of various SSL tasks with respect to DDI and DTI predictions, because they are key stages and play important roles in various applications of drug discovery. Simultaneously, DDI and DTI predictions are treated as link predictions in homogeneous and heterogeneous networks, respectively. Therefore, DDI and DTI predictions can systematically demonstrate the performance of various kinds of SSL tasks and joint strategies. According to the guidance of KGE_NFM [5], we design the following two experimental scenarios. Warm start predictions: Given a set of drugs and their known DTIs, we aim to predict other potential interactions between these drugs. All the known interactions are positive samples, and an equal number of negative samples are randomly selected from the unknown interactions. The positive and negative samples are split into a training set (90\%) and a testing set (10\%). In this situation, the training set may include drugs and targets contained in the test set. The same experimental setting as DTI predictions are used for DDI predictions. In the experimental scenarios, we compare the differences among various SSL tasks for DDI and DTI predictions and draw a conclusion on which SSL task and combination strategies can generate the best performance. Cold start for drugs: In real drug discovery, it is more important and challenging to predict potential targets and drugs that may interact with newly discovered chemical compounds. In other words, the test set contains drugs that are unseen in the training set. Here, we randomly select 5\% drugs, and then all DTI and DDI pairs associated with these drugs are treated as the test set. This scenario aims to validate the conclusions that are found in the warm start predictions.

\section{Data availability}

The details of original network data and prepossessed input data demo can be downloaded from https://github.com/pengsl-lab/MSSL2drug.git.

\section{Code availability}

The source code can be found at: https://github.com/pengsl-lab/MSSL2drug.git.

\section{Acknowledgement}

This work was supported by NSFC Grants U19A2067, 61772543, 81973244; Science Foundation for Distinguished Young Scholars of Hunan Province (2020JJ2009); National Key R\&D Program of China 2017YFB0202602, 2018YFC0910405, 2017YFC1311003, 2016YFC1302500; Science Foundation of Changsha Z202069420652, kq2004010; JZ20195242029, JH20199142034; The Funds of State Key Laboratory of Chemo/Biosensing and Chemometrics and Peng Cheng Lab; Hunan Provincial Innovation Foundation For Postgraduate. 


\section{Author contributions}

X.W. and Y.C. conceived the original idea and developed the code for the core algorithm. S. P designed the experiment and wrote the initial version of the manuscript. F.L. analyzed the experimental data and edited this manuscript. Y.Y constructed the biomedical network data. All authors reviewed and approved the final manuscript.

Competing interests: The authors declare no competing interests.

\section{References}

[1] Dickson, M. \& Gagnon, J. P. Key factors in the rising cost of new drug discovery and development. Nat. Rev. Drug Discov. 3, 417-429 (2004).

[2] Scannell, J. , Blanckley, A., Boldon, H., \& Warrington, B. Diagnosing the decline in pharmaceutical R\&D efficiency. Nat. Rev. Drug Discov. 11, 191-200 (2012).

[3] Shen, W. X. et al. Out-of-the-box deep learning prediction of pharmaceutical properties by broadly learned knowledge-based molecular representations. Nat. Mach. Intell. 3, 334-343 (2021).

[4] Chen, D. et al. Algebraic graph-assisted bidirectional transformers for molecular property prediction. Nat. Commun. 12, 1-9 (2021).

[5] Ye, Q. at al. A unified drug-target interaction prediction framework based on knowledge graph and recommendation system. Nat. Commun. 12, 1-12 (2021).

[6] Luo, Y. at al. A network integration approach for drug-target interaction prediction and computational drug repositioning from heterogeneous information. Nat. Commun. 8, 1-13 (2017).

[7] Cheng, F. at al. Network-based approach to prediction and population-based validation of in silico drug repurposing. Nat. Commun. 9, 1-12 (2018).

[8] Zheng, S. at al. Predicting drug-protein interaction using quasi-visual question answering system. Nat. Mach. Intell. 2, 134-140 (2020).

[9] Liu, R., Wei, L., \& Zhang, P. A deep learning framework for drug repurposing via emulating clinical trials on real-world patient data. Nat. Mach. Intell. 3, 68-75 (2021).

[10] Ryu, J., Kim, H., \& Lee, S. Deep learning improves prediction of drug-drug and drug-food interactions. Proc. Natl. Acad. Sci. U.S.A. 115, E4304-E4311 (2018).

[11] Cheng, F., Kovács, I. A., \& Barabási, A. L. Network-based prediction of drug combinations. Nat. Commun. 10, 1-11 (2019).

[12] Jiménez-Luna, J., Grisoni, F., \& Schneider, G. Drug discovery with explainable artificial intelligence. Nat. Mach. Intell. 2, 573-584 (2020).

[13] Zeiler, M. D., \& Fergus, R. Visualizing and understanding convolutional networks. In Proceedings of the 13th European Conference on Computer Vision 818-833 (Springer, 2014).

[14] Hochreiter, S., \& Schmidhuber, J. Long short-term memory. Neural Comput 9, 1735-1780 (1997).

[15] Kipf, T. N., \& Welling, M. Semi-supervised classification with graph convolutional networks. In Proceedings of the 4th International Conference on 
Learning Representations (OpenReview.net, 2017).

[16] Veličković, P., Cucurull, G., Casanova, A., Romero, A., Lio, P., \& Bengio, Y. Graph Attention Networks. In Proceedings of the 5th International Conference on Learning Representations (OpenReview.net, 2018).

[17] Hamilton, W. L., Ying, R., \& Leskovec, J. Inductive representation learning on large graphs. In Proceedings of the 31st International Conference on Neural Information Processing Systems 1025-1035 (MIT Press, 2017).

[18] Zitnik, M., Agrawal, M., \& Leskovec, J. Modeling polypharmacy side effects with graph convolutional networks. Bioinformatics 34, i457-i466 (2018).

[19] Ma, T., Xiao, C., Zhou, J., \& Wang, F. Drug similarity integration through attentive multi-view graph auto-encoders. In Proceedings of the 27th International Joint Conference on Artificial Intelligence 3477-3483 (Morgan Kaufmann, 2018).

[20] Gysi, D. M. et al. Network medicine framework for identifying drug-repurposing opportunities for COVID-19. Proc. Natl. Acad. Sci. U.S.A. 118 (2021).

[21] Wang, Z., Zhou, M., \& Arnold, C. Toward heterogeneous information fusion: bipartite graph convolutional networks for in silico drug repurposing. Bioinformatics 36, i525-i533 (2020).

[22] He, K., Fan, H., Wu, Y., Xie, S., \& Girshick, R. Momentum contrast for unsupervised visual representation learning. In Proceedings of the 33th IEEE/CVF Conference on Computer Vision and Pattern Recognition 9729-9738 (IEEE, 2020).

[23] Grill, J. B. et al. Bootstrap your own latent: A new approach to self-supervised learning. In Proceedings of the 34th Conference on Neural Information Processing Systems 33, 21271-21284 (MIT Press, 2020).

[24] Devlin, J., Chang, M. W., Lee, K., \& Toutanova, K. BERT: Pre-training of deep bidirectional transformers for language understanding. In Proceedings of the Conference of the North American Chapter of the Association for Computational Linguistic 4171-4186 (NAACL, 2019)

[25] Brown, T. B. et al. Language models are few-shot learners. In Proceedings of the 34th Conference on Neural Information Processing Systems 33, 1877-1901 (MIT Press, 2020).

[26] Pham, T.H., Qiu, Y., Zeng, J., Xie, L., \& Zhang, P. A deep learning framework for high-throughput mechanism-driven phenotype compound screening. Nat Mach Intell 3, 247-257 (2021).

[27] Wang, Y., Min, Y., Chen, X., \& Wu, J. Multi-view Graph Contrastive Representation Learning for Drug-Drug Interaction Prediction. In Proceedings of the 30th Web Conference 2921-2933 (ACM, 2021).

[28] Wang, X. et al. DeepR2cov: deep representation learning on heterogeneous drug networks to discover anti-inflammatory agents for COVID-19. Brief. Bioinformatics 22, 1-14 (2021).

[29] Zeng, X. at al. Network-based prediction of drug-target interactions using an arbitrary-order proximity embedded deep forest. Bioinformatics 36, 2805-2812 (2020). 
[30] Rong, Y. et al. Self-supervised graph transformer on large-scale molecular data. In Proceedings of the 34th Conference on Neural Information Processing Systems 33, 12559-12571 (MIT Press, 2020).

[31] Wang, X. et al. BioERP: biomedical heterogeneous network-based selfsupervised representation learning approach for entity relationship predictions. Bioinformatics 37, 4793-4800 (2021).

[32] $\mathrm{Hu}, \mathrm{W}$. et al. Strategies for pre-training graph neural networks. In Proceedings of the 8th International Conference on Learning Representations (OpenReview.net, 2020).

[33] Zeng, X. at al. Target identification among known drugs by deep learning from heterogeneous networks. Chem. Sci. 11, 1775-1797 (2020).

[34] Costa, L. D. F. et al. Analyzing and modeling real-world phenomena with complex networks: a survey of applications. Adv. Phys. 60, 329-412 (2011).

[35] Peng, Z., Dong, Y., Luo, M., Wu, X. M., \& Zheng, Q. Self-supervised graph representation learning via global context prediction. Preprint at https://arxiv.org/ abs/2003.01604 (2020).

[36] Watts, D. J. Networks, dynamics, and the small-world phenomenon. Am. J. Sociol. 105, 493-527 (1999).

[37] Taylor, W. L. "Cloze procedure": a new tool for measuring readability. Journalism Quarterly 30, 415-433 (1953).

[38] Weininger, D. SMILES, a chemical language and information system. 1. Introduction to methodology and encoding rules. J Chem Inf Model 28, 31-36 (1988).

[39] Vilar, S. et al. Similarity-based modeling in large-scale prediction of drug-drug interactions. Nat. Protoc. 9, 2147-2163 (2014).

[40] Gotoh, O. An improved algorithm for matching biological sequences. J. Mol. Biol. 162, 705-708 (1982).

[41] Ni, P. at al. Constructing disease similarity networks based on disease module theory. IEEE/ACM Trans. Comput. Biol. Bioinform. 17, 906-915 (2018).

[42] Liu, P., Qiu, X., \& Huang, X. Adversarial multi-task learning for text classification. In Proceedings of the 55th Annual Meeting of the Association for Computational Linguistics 1-10 (ACL, 2017).

[43] Bousmalis, K., Trigeorgis, G., Silberman, N., Krishnan, D., \& Erhan, D. Domain separation networks. In Proceedings of the 30th Conference on Neural Information Processing Systems 29, 343-351 (2016).

[44] Collobert, R., \& Weston, J. A unified architecture for natural language processing: Deep neural networks with multitask learning. In Proceedings of the 25th International Conference on Machine Learning 160-167 (ACM, 2008).

[45] Glorot, X., \& Bengio, Y. Understanding the difficulty of training deep feedforward neural networks. In Proceedings of the 13th International Conference on Artificial Intelligence and Statistics 249-256 (JMLR, 2010).

[46] Kingma, D. P., \& Ba, J. Adam: A method for stochastic optimization. In Proceedings of the 3rd International Conference for Learning Representations (OpenReview.net, 2015). 


\section{Supplementary Files}

This is a list of supplementary files associated with this preprint. Click to download.

- SupplementaryMaterials.pdf

- PengCSSCflat.pdf 